\title{
Salivary Cortisol Concentration Changes After Epidural Steroid Injection
}

Jin Young Chon, MD, PhD, and Ho Sik Moon, MD

From: Department of
Anesthesiology and Pain
Medicine, The Catholic
University of Korea, Seoul,
Korea
Address Correspondence:
Ho Sik Moon, MD
Department of Anesthesiology
and Pain Medicine,
Yeouido St. Mary's Hospital,
College of Medicine,
The Catholic University of
Korea 10 63-ro, Yeongdeungpo-
gu, Seoul 150-713, South Korea.
E-mail:
mhsjshcat@catholic.ac.kr
Disclaimer: There was no
external funding in the

egenerative spinal diseases such as herniated intervertebral disc and spinal stenosis are among the most common musculoskeletal diseases. These disorders often respond well to exercise about the safety of steroids. of triamcinolone.

Setting: Outpatient follow-up. concentrations, and NRS scores. stress on cortisol levels. spinal stenosis.

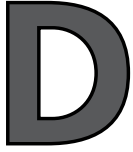

Background: Epidural steroid injections (ESI) are therapeutically useful for the treatment of herniated intervertebral discs and spinal stenosis. However, there is a lack of evidence-based data

Objectives: The aim of this study was to determine the period of hypothalamus-pituitary-adrenal (HPA) axis suppression by salivary cortisol measurements after a single epidural injection of $40 \mathrm{mg}$

\section{Study Design: Clinical observational pilot study.}

Methods: In this study, 8 patients with lumbar intervertebral disc herniation or spinal stenosis were enrolled. All patients had received ESI (triamcinolone $40 \mathrm{mg}$ ) under C-arm guidance. Salivary cortisol concentrations were assessed between 8:00 am and 9:00 am; both before and after ESI (Day 0 [the day prior to injection], 1, 3, 5, 7, 14, 21, 28). Additionally, body weight, blood pressure, 0-10 numeric rating scale (NRS), and fasting blood sugar levels were evaluated.

Results: HPA axis suppression was observed in all patients for $19.9 \pm 6.8$ days after ESI (salivary cortisol $<0.1 \mu \mathrm{g} / \mathrm{mL}$ ). In total, 5 of 8 patients reached baseline salivary cortisol concentrations within the clinical trial period. The time taken to reach baseline concentrations after ESI was 19.4 \pm 8.3 days. The period of HPA axis suppression was not correlated with baseline salivary cortisol

Limitations: Adrenal insufficiency cannot be confirmed by simple salivary cortisol measurements. Furthermore, the period studied was too short for all patients' HPA axis function to reach the baseline value. Finally, the study design does not allow for the confounding effects of pain and

Conclusions: HPA axis function was suppressed after ESI until Day 21 and returned to the normal range after $19.9 \pm 6.8$ days. Therefore, we suggest that the minimal interval between ESI treatments should be at least one month. In addition, we report that salivary cortisol measurements are very useful diagnostic predictors of HPA function.

Key words: Epidural injections, salivary, steroids, HPA, complications, cortisol, herniated discs,

Pain Physician 2012; 15:461-466 or physical therapy, or can resolve spontaneously. Alternatively, these disorders may be treated with interventional pain management or surgery. Although epidural steroid injections (ESI) are often used as 
interventional pain management, there is a lack of evidence-based data regarding the pharmacologic properties, effects, and safety of this therapy. In fact, complications of corticosteroid administration have been exaggerated, and the actual incidence of adverse effects from ESI is low (1). However, there are a few rebuttable cases. One of the most common side effects of iatrogenic steroid administration is secondary suppression of the hypothalamus-pituitary-adrenal (HPA) axis. The gold standard test for evaluating HPA axis function is adrenocorticotropic hormone (ACTH) stimulation test using $250 \mu \mathrm{g}$ synthetic ACTH (2). This method can accurately diagnose HPA suppression or adrenal insufficiency, but is invasive, uncomfortable for patients, and in a few cases requires patients to visit the hospital for follow-up examinations.

There are 2 forms of cortisol: free cortisol and protein-bound cortisol in the blood (3-5). Free cortisol is known to be biologically active and can be measured by a 24-hour collection of urine or salivary cortisol (5). Salivary cortisol measurements have the advantages of being simple and noninvasive. They provide a highly accurate diagnosis and are associated with high patient compliance (4-7). A Food and Drug Administrationcleared enzyme-linked immunosorbent assay (ELISA) is commercially available (8). Hence, we could analyze the effect of time on HPA axis suppression by measuring salivary cortisol concentrations after ESI. We hypothesized that salivary cortisol measurements may be used as an indication of early complications of ESI.

Here, we performed a pilot clinical observational study. The primary outcome measure was the period of HPA axis suppression by salivary cortisol measurements after single ESI. The secondary outcome measure was the relationship between the period of HPA axis suppression, initial pain intensity, and baseline salivary cortisol concentration.

\section{Methods}

\section{Patients}

The Institutional Review Board of the Catholic University of Korea approved the protocols for this study. All patients provided written informed consent to participate in this study. All 8 patients were diagnosed with lumbar intervertebral disc herniation or spinal stenosis according to their symptoms and magnetic resonance imaging (MRI) findings. At inclusion, medical histories were taken and physical examinations performed. Inclusion criteria required that patients met the American
Society of Anesthesiologists Physical Status 1 classification, were over 20 years old, were receiving ESI for pain management, and had their hospital appointments in the early morning (8:00 to 9:00). Exclusion criteria included coagulopathy, infection at the procedure site, allergy to corticosteroids, pregnancy, all endocrinology diseases, history of corticosteroid treatment in the preceding year, chronic medical illnesses (diabetes, hypertension, heart failure, renal disease, liver disease, etc.), sleep disturbance, taking herbal medicine or contraceptives, severe stress in the preceding month, and diseases of the oral cavity. None of the subjects consumed alcohol 12 hours before sampling or food one hour before sampling.

\section{Intervention}

All patients received a single injection of $40 \mathrm{mg}$ triamcinolone acetonide in $6 \mathrm{~mL}$ mepivacaine $\mathrm{HCl}$ epidurally. All procedures were performed under C-arm guidance. Intravascular injection was excluded through use of contrast medium. After epidural injection, all patients received a single analgesic (celecoxib $200 \mathrm{mg}$ ) as rescue medication.

\section{Measurement}

Before epidural block (Day 0), body weight, blood pressure, $0-10$ numeric rating scale (NRS) (0, no pain; 10, worst pain imaginable), fasting blood sugar levels, and salivary cortisol levels were assessed. Saliva was obtained from the participants by using a commercially available cotton sampling device, Salivette (Salimetrics, State College, PA). During the 28-day study period, salivary samples were collected on Days $1,3,5,7,14$, 21, and 28 after ESI. Patients were instructed to rinse their mouth thoroughly with water 10 minutes before sample collection between 8:00 am and 9:00 am. They stored their saliva sample in a freezer compartment. Salivary samples were frozen at or below $-20^{\circ}$ within 4 hours after collection (9). On Day 28, the patients revisited our pain clinic with their samples in a cooler. At that time, body weight, blood pressure, NRS, and fasting blood sugar level were assessed. Salivary cortisol levels were measured by ELISA using the VersaMax ELISA Microplate Reader (Molecular Devices, Sunnyvale, CA).

\section{Statistical Analyses}

All data are presented as means \pm standard deviation or frequency. Salivary cortisol level data measured across time were analyzed using paired t-tests. Similarly, comparison of NRS, body weight, fasting blood 
sugar level, and blood pressure between before ESI and Day 28 were performed using paired t-tests. Correlation analysis between NRS and changes in salivary cortisol levels, and between the time taken to reach normal range $(\geq 0.1 \mu \mathrm{g} / \mathrm{mL})$ and other values were performed by Pearson's correlation analysis. HPA-suppressed intervals were calculated by the interpolation method, i.e., we constructed new data points within the range of a discrete set of known data points (Day 0, Day 1, Day 3, Day 5, Day 7, Day 14, Day 21, and Day 28). A P-value of < 0.05 was considered significant. Statistical analyses were performed using the statistical package SAS 9.2 (SAS Institute, Inc., Cary, NC).

\section{Results}

We included 6 lumbar intervertebral disc herniation and 2 spinal stenosis patients. All patients were steroid-naïve. They maintained their regular lifestyle, waking up between 7:00 am and 8:00 am and going to bed between 10:00 pm and 11:00 pm. In demographic and baseline data, significant differences between Day 0 and Day 28 were not observed, with the exception of NRS. Compared with NRS before ESI, NRS was decreased on Day 28 , from $6.6 \pm 1.5$ to $3.6 \pm 1.7(P<0.05)$ (Table 1$)$. Only 2 of the patients took the offered analgesic; one patient took $600 \mathrm{mg} / \mathrm{d}$-> $600 \mathrm{mg}$ celecoxib for 3 days, while another took $1000 \mathrm{mg} / \mathrm{d}$-> $1000 \mathrm{mg}$ celecoxib for 4 days.

All patients followed the protocol, collecting and storing saliva samples as requested. No study patient revisited our clinic for ESI during the clinical trial period, or take any medicine that could affect salivary cortisol concentrations. All patients' HPA axis were suppressed for $19.9 \pm 6.8$ days after ESI (salivary cortisol < $0.1 \mu \mathrm{g} / \mathrm{mL}$ ). In total, 5 patients reached baseline salivary cortisol concentrations within the clinical trial period. The time taken to reach baseline values was $19.4 \pm 8.3$ days after ESI in these 5 patients (Table 2 and Fig. 1).

The periods of HPA axis suppression were not correlated with other variables of baseline salivary cortisol concentration, and NRS score (Pearson correlation coefficient, $r<0.8 ; P>0.05)$. The salivary cortisol concentration difference during the first day and baseline NRS score was not significantly correlated $(r<0.8, P>0.05)$.

\section{Discussion}

Cortisol is a steroid hormone that is released by cells of the adrenal cortex stimulated by ACTH (10). Up to $95 \%$ of the secreted cortisol is bound to protein such as cortisol binding globulin (CBG) or albumin, while the remaining cortisol is secreted unbound, i.e., in the free form, which is thought to be biologically active
Table 1. Demographic data and baseline values for patients $(n=8)$.

\begin{tabular}{|l|c|}
\hline \multicolumn{2}{|l|}{ Clinical Characteristics } \\
\hline Age (yr) & $47.4 \pm 21.8$ \\
\hline Gender (M/F) & $4 / 4$ \\
\hline Weight (kg), Day 0 & $66.9 \pm 14.4$ \\
\hline Weight (kg), Day 28 & $67.0 \pm 14.4$ \\
\hline SBP (mm Hg), Day 0 & $129.4 \pm 12.8$ \\
\hline SBP (mm Hg), Day 28 & $128.1 \pm 6.2$ \\
\hline DBP (mm Hg), Day 0 & $82.8 \pm 11.1$ \\
\hline DBP (mm Hg), Day 28 & $81.9 \pm 6.8$ \\
\hline NRS, Day 0 & $6.6 \pm 1.5$ \\
\hline NRS, Day 28 & $3.6 \pm 1.7 *$ \\
\hline FBS (g/mL), Day 0 & $101.9 \pm 14.7$ \\
\hline FBS (g/mL), Day 28 & $98.4 \pm 12.7$ \\
\hline
\end{tabular}

All values are mean \pm SD or number. Day $0=$ Day before epidural steroid injection; Day $28=$ Day after epidural steroid injection. DBP $=$ Diastolic blood pressure; FBS = Fasting blood sugar level; NRS = Numeric rating scale ( 0 no pain, 10 worst pain imaginable); $\mathrm{SBP}=$ Systolic blood pressure. ${ }^{*} P<0.05$ for differences between Day 0 and Day 28 .
Table 2. Changes of salivary cortisol concentration over 28 days.

\begin{tabular}{|l|c|}
\hline Variables & Mean \pm SD \\
\hline Day 0 $(\mu \mathrm{g} / \mathrm{mL})$ & $0.16 \pm 0.06$ \\
\hline Day 1 & $0.03 \pm 0.02^{\star}$ \\
\hline Day 3 & $0.04 \pm 0.03^{\star}$ \\
\hline Day 5 & $0.04 \pm 0.02^{\star}$ \\
\hline Day 7 & $0.04 \pm 0.03^{\star}$ \\
\hline Day 14 & $0.07 \pm 0.07^{\star}$ \\
\hline Day 21 & $0.14 \pm 0.12$ \\
\hline Day 28 & $0.24 \pm 0.12$ \\
\hline HPA suppressed period (days) & $19.9 \pm 6.8$ \\
\hline
\end{tabular}

Day 0 = Day before epidural steroid injection; Day $28=$ Day after epidural steroid injection; HPA suppressed period indicates the interval of early morning salivary cortisol concentration is beyond $0.1 \mu \mathrm{g} / \mathrm{ml}$. ${ }^{*} P<0.05$ compared with Day 0 . 


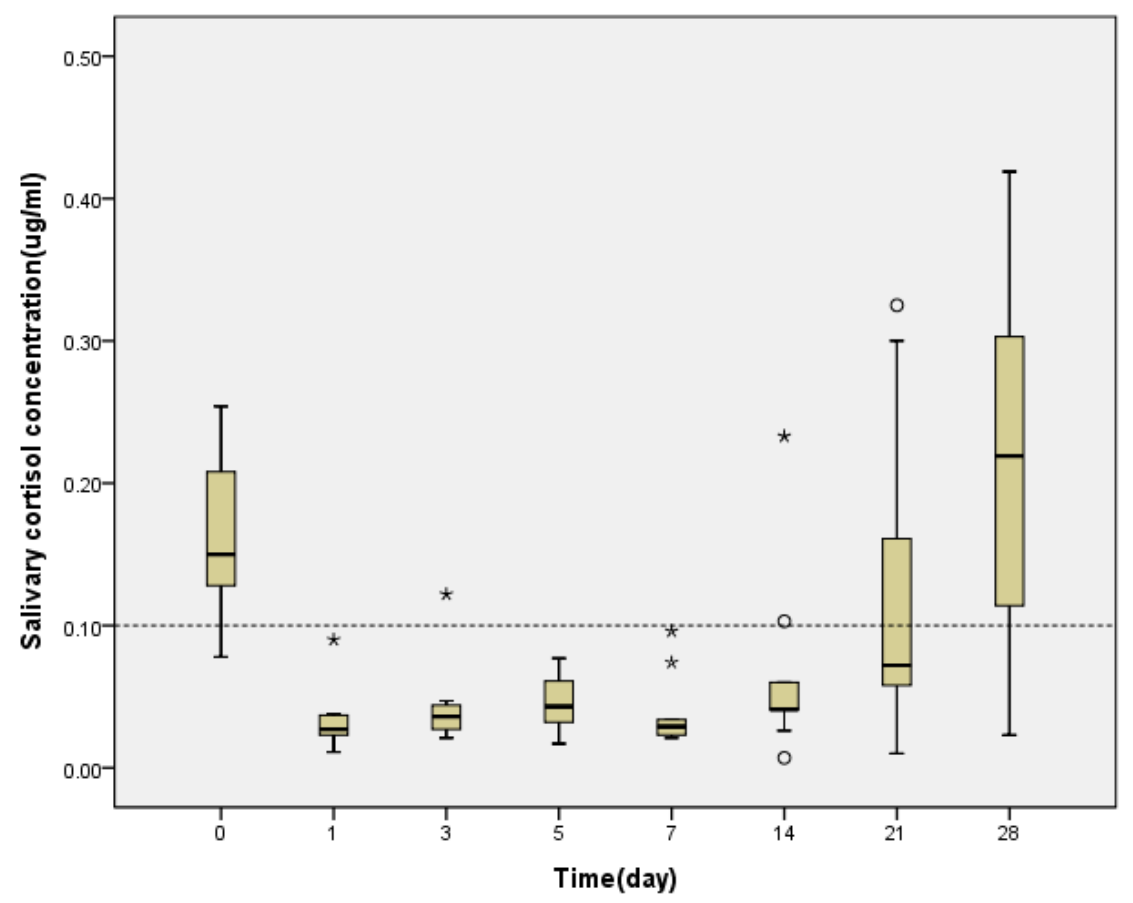

Fig. 1. Salivary cortisol concentration changes over 28 days. The time course and response pattern (mean \pm SD) in salivary cortisol. After epidural steroid injection, salivary cortisol concentrations of all patients were initially suppressed, before returning to normal levels. However, salivary cortisol concentrations returned to the baseline value within 28 days in only 5 patients.

$(4,9,10)$. Free cortisol levels can be assessed by 24-hour urinary free cortisol or salivary cortisol concentrations (4). Recently, interest in the use of salivary cortisol has increased because salivary cortisol measurements have a number of significant advantages: first, they are accurate and representative of circulating levels, and second, sample collection is simple and cost effective $(3,5$, 7-11). The primary aim of this study was to determine the duration of HPA axis suppression after ESI. Ordinarily, this requires frequent hospital visits and invasive blood tests at set times. In reality, patient compliance to this regimen is low, and it is therefore difficult to design a study protocol based on the traditional approach. Therefore, in this prospective clinical observational study, we used salivary cortisol to assess HPA axis function.

The study of salivary cortisol concentrations is significantly limited by several factors. Medications that affect the salivary cortisol level act via several different mechanisms: They may affect the subjective experience of stress, threat, or pain; they may act as agonists or antagonists of specific HPA secretagogues; they may affect synthesis or release of cortisol from the adrenal gland; they may have physiological effects on the HPA network system or affect the transport of serum constituents into oral fluid; they may also affect the composition of saliva, CBG levels, or binding to cortisol by specifically cross-reacting with anti-cortisol antibodies, or nonspecific interference in relation to cortisol antibody binding; and they may be administered via intranasal or oral routes as either an inhalant or topical gel (12). When the current study was designed, we excluded patients receiving any medications with the above-mentioned mechanism. Triamcinolone has a very low crossreactivity in the assay for salivary cortisol. The reported cross-reactivity of triamcinolone is $0.09 \%$, prednisolone $0.57 \%$, cortisone $0.13 \%$, dexamethasone $19.20 \%$, corticosterone $0.21 \%$, progesterone $0.02 \%$, and testosterone $0.01 \%$ (9). Therefore, we excluded the possibility that cross-reactivity of triamcinolone affected salivary cortisol levels while analyzing the current results.

The precision of salivary cortisol measurements using commercial kits is essential in these studies. The diagnostic value of salivary cortisol for HPA axis function has received significant recent attention (3-8). Analysis of cortisol levels in 174 patients using a commercial sali- 
vary cortisol enzyme immunoassay kit revealed that mean intra-assay coefficients of variations (CVs) were $\leq 2.5 \%$, mean inter-assay CVs were $\leq 2.8 \%$, and priori criteria for intra- and inter- assay CVs were $\leq 4 \%$ and $\leq$ $7 \%$ respectively (10). Gozansky et al (4) found that salivary cortisol measurements using commercial kits could be used for the assessment of dynamic HPA axis activity instead of serum total cortisol. Furthermore, consideration of intra-subject and inter-subject changes in CBG was not deemed necessary.

Steroids have been widely used in medicine for a long time. These drugs have several benefits for the treatment of various diseases, including their antiinflammatory, immunosuppressive, bone marrow stimulatory, and growth stimulatory effects. However, steroids can cause many adverse effects, including elevated blood pressure, increased risk of cardiovascular disease, acne, liver damage, reduced sexual function, increased body hair, temporary decrease in menstrual cycles, epiphyseal fusion, accelerated bone maturation, and various psychiatric effects. Adverse effects specifically related to epidural injection of steroids commonly include suppression of glucocorticoid secretion (13).

When epidural triamcinolone acetate $(80 \mathrm{mg}$ weekly for 3 weeks) was injected in 14 patients, dramatic acute and chronic suppression of the HPA axis was observed by measuring plasma ACTH and by evaluating the cortisol response to cosyntropin. Median suppression was less than one month, and all patients had recovered by 3 months (14). In the present study, the mean suppression period by salivary cortisol measurements was $19.9 \pm 6.8$ days, although cosyntropin stimulating tests were not performed. Since the parameters of our study are different from those of the previous study, direct comparison between the results of both studies cannot be drawn. However, the duration of HPA axis suppression is considered to be dose dependent. Furthermore, the duration of HPA axis suppression may be influenced by the site of injection and the different corticosteroid preparations (15-17). Guidelines for frequency, dosage, and timing of repeat ESIs have not yet been suggested, primarily because of a lack of evidence-based reports on the subject (18).

Adrenal cortisol levels have circadian rhythm, and are also affected by age, seasonal, and gender differences (7). Adrenal cortisol concentrations peak in the morning, and are the lowest around midnight $(19,20)$. Therefore, morning salivary cortisol measurements are useful diagnostic indicators of adrenal suppression. Conversely, late-night salivary cortisol measurements are useful to diagnose Cushing disease (5). Although serum cortisol levels were not found to decline in an agerelated pattern, salivary cortisol levels were not found to alter with age in Korean populations (20). Salivary cortisol levels are the highest in February, March, and April, and the lowest in July and August $(21,22)$. In this study, all pateints were studied during November and December to exclude seasonal variation. There are also gender differences in cortisol secretion in chronic pain syndromes, with salivary cortisol concentrations lower in men than in women at noon and 6:00 pm. However, there are no significant sex differences in healthy controls (23).

Several limitations of this pilot study should be considered when interpreting the results. First, adrenal insufficiency cannot be confirmed by simple salivary cortisol measurements (5). For adrenal insufficiency, an ACTH stimulation test is needed. As the primary focus of this study was to ascertain changes of HPA axis function and monitor trends in these changes, we used salivary measurements in this pilot investigation. Second, the study period was short, and therefore, analyses were limited. For example, we do not know at what time salivary cortisol concentrations reached to the initial value. Rather, we only determined that these concentrations returned to initial levels in 5 of 8 patients. Third, we did not exclude the potential effect of pain and stress on cortisol levels.

This study is consistent with prior studies showing that HPA axis suppression is altered after ESI. The use of a simple, noninvasive, cost-effective diagnostic tool like salivary cortisol measurements, will allow further research on the effects of ESI to be undertaken. Additional studies using extended treatment intervals, varied steroid dosages, and allowable periods of ESI are warranted.

\section{Conclusion}

In summary, our study demonstrates that the HPA axis function of all patients was suppressed after ESI, but returned to the normal range after $19.9 \pm 6.8$ days. Therefore, we suggest the minimal interval between ESIs should be one month. Salivary cortisol measurements are a very useful diagnostic indicator of HPA function. 


\section{References}

1. McGrath JM, Schaefer MP, Malkamaki DM. Incidence and characteristics of complications from epidural steroid injections. Pain Med 2011; 12:726-731.

2. Neary N, Nieman L. Adrenal insufficiency: Etiology, diagnosis and treatment. Curr Opin Endocrinol Diabetes Obes 2010; 17:217-223.

3. Sakihara S, Kageyama K, Oki Y, Doi M, Iwasaki Y, Takayasu S, Moriyama T, Terui K, Nigawara T, Hirata $Y$, Hashimoto $\mathrm{K}$, Suda T. Evaluation of plasma, salivary, and urinary cortisol levels for diagnosis of Cushing's syndrome. Endocr J 2010; 57:331-337.

4. Gozansky WS, Lynn JS, Laudenslager ML, Kohrt WM. Salivary cortisol determined by enzyme immunoassay is preferable to serum total cortisol for assessment of dynamic hypothalamic--pituitary--adrenal axis activity. Clin Endocrinol (Oxf) 2005; 63:336-341.

5. Raff $\mathrm{H}$. Utility of salivary cortisol measurements in Cushing's syndrome and adrenal insufficiency. J Clin Endocrinol Metab 2009; 94:3647-3655.

6. Carrasco CA, Garcia M, Goycoolea M, Cerda J, Bertherat J, Padilla O, Meza D, Wohllk N, Quiroga T. Reproducibility and performance of one or two samples of salivary cortisol in the diagnosis of Cushing's syndrome using an automated immunoassay system. Endocrine 2012; 41:487-493.

7. Tornhage CJ. Salivary cortisol for assessment of hypothalamic-pituitary-adrenal axis function. Neuroimmunomodulation 2009; 16:284-289.

8. Raff H, Homar PJ, Skoner DP. New en- zyme immunoassay for salivary cortisol. Clin Chem 2003; 49:203-204.

9. Salimetrics. High Sensitivity Salivary Cortisol Enzyme Immunoassay Kit. March 2011. www.salimetrics.com/assets/documents/products-and-services/ salivary-assays/Cortisol\%2oKit\%2olnsert.pdf

10. Hanneman SK, Cox CD, Green KE, Kang $\mathrm{DH}$. Estimating intra- and inter-assay variability in salivary cortisol. Biol Res Nurs 2011; 13:243-250.

11. Aardal E, Holm AC. Cortisol in saliva-reference ranges and relation to cortisol in serum. Eur ] Clin Chem Clin Biochem 1995; 33:927-932.

12. Granger DA, Hibel LC, Fortunato CK, Kapelewski $\mathrm{CH}$. Medication effects on salivary cortisol: Tactics and strategy to minimize impact in behavioral and developmental science. Psychoneuroendocrinology 2009; 34:1437-1448.

13. Bogduk N. Epidural steroid injections. In: Fishman S, Ballantyne J, Rathmell (eds). Bonica's Management of Pain, 4th edition. Lippincott Williams \& Wilkins, Baltimore, 2010, pp 1423-1437.

14. Kay J, Findling JW, Raff H. Epidural triamcinolone suppresses the pituitaryadrenal axis in human subjects. Anesth Analg 1994; 79:501-505.

15. $\mathrm{L}$ azarevic MB, Skosey JL, Djordjevic-Denic G, Swedler WI, Zgradic I, Myones BL. Reduction of cortisol levels after single intra-articular and intramuscular steroid injection. Am J Med 1995; 99:370-373.

16. Maillefert JF, Aho S, Piroth-Chatard C, Tavernier C. Cortisol levels after single local steroid injection. Am J Med 1996; 100:586-587.

17. Morkane C, Gregory JW, Watts P, Warner JT. Adrenal suppression following intralesional corticosteroids for periocular haemangiomas. Arch Dis Child 2011; 96:587-589.

18. Novak S, Nemeth WC. The basis for recommending repeating epidural steroid injections for radicular low back pain: A literature review. Arch Phys Med Rehabil 2008; 89:543-552.

19. Arlt W. Disorders of the adrenal cortex. In: Longo D, Fauci A, Kasper D, Hauser $S$, Jameson J, Loscalzo J (eds). Harrison's Principles of Internal Medicine, 18th edition. McGraw-Hill, New York, 2011, pp 2940-2961.

20. Ahn RS, Lee YJ, Choi JY, Kwon HB, Chun SI. Salivary cortisol and DHEA levels in the Korean population: Age-related differences, diurnal rhythm, and correlations with serum levels. Yonsei Med J 2007; 48:379-388.

21. Persson R, Garde AH, Hansen AM, Osterberg K, Larsson B, Orbaek P, Karlson $B$. Seasonal variation in human salivary cortisol concentration. Chronobiol Int 2008; 25:923-937.

22. Walker BR, Best R, Noon JP, Watt GC, Webb DJ. Seasonal variation in glucocorticoid activity in healthy men. J Clin Endocrinol Metab 1997; 82:4015-4019.

23. Turner-Cobb JM, Osborn M, da Silva $L$, Keogh E, Jessop DS. Sex differences in hypothalamic-pituitary-adrenal axis function in patients with chronic pain syndrome. Stress 2010; 13:292-300. 